\title{
Paris saponin II inhibits human ovarian cancer cell-induced angiogenesis by modulating NF- $\mathrm{KB}$ signaling
}

\author{
MEI YANG ${ }^{1,2,3}$, JUAN ZOU ${ }^{4}$, HONGMEI ZHU ${ }^{1,3,5}$, SHANLING LIU ${ }^{1,3,5}$, \\ $\mathrm{HE} \mathrm{WANG}^{1,2,3}$, PENG BAI ${ }^{6^{*}}$ and XUE XIAO ${ }^{1,2,3,7^{*}}$
}

\begin{abstract}
${ }^{1}$ Department of Obstetrics and Gynecology, West China Second University Hospital, Sichuan University; ${ }^{2}$ Laboratory of Genetics, West China Institutes for Women and Children's Health, West China Second University Hospital, Sichuan University; ${ }^{3}$ Key Laboratory of Obstetrics and Gynecologic and Pediatric Diseases and Birth Defects of Ministry of Education, West China Second University Hospital, Sichuan University; ${ }^{4}$ Department of Pathology, West China Second University Hospital, Sichuan University; ${ }^{5}$ Laboratory of Cell and Gene Therapy, West China Institutes of Women and Children's Health, West China Second University Hospital, Sichuan University; ${ }^{6}$ West China School of Preclinical and Forensic Medicine, Sichuan University, Chengdu, Sichuan 610041, P.R. China; ${ }^{7}$ Department of Pathology,

The University of Texas M.D. Anderson Cancer Center, Houston, TX, USA
\end{abstract}

Received October 9, 2014; Accepted December 29, 2014

DOI: $10.3892 /$ or.2015.3836

\begin{abstract}
The clinical applications of Rhizoma paridis in traditional Chinese medicine are well known. However, the therapeutic potential of Rhizoma paridis and its active component such as Paris saponin I (polyphyllin D) and Paris saponin II (PSII) (formosanin C) in cancer treatments have not yet been fully explored. Recent studies have demonstrated that PSII and chemoagents exhibit comparable inhibitory affects against human ovarian cancer cell growth. Since NF- $\kappa$ B, a ubiquitous transcription factor that plays an important role in cancer biology, is often associated with gynecological cancers, in the present study, we evaluated the possibility that PSII modulates NF- $\mathrm{B}$ activity and VEGF-mediated angiogenesis and elucidated the molecular mechanisms underlying such effects. We assessed the effects of PSII on NF- $\kappa \mathrm{B}$ activity in SKOV3 tumor cells and on tumor cell induced-angiogenesis using standardized angiogenesis in vitro, ex vivo and in vivo assays, western blot analysis and kinase assay. We also assessed the effect of the super-engineered repressor of $\operatorname{IkB} \alpha$ and its effect, in combination with PSII treatment on tumor growth and
\end{abstract}

Correspondence to: Professor Xue Xiao, Department of Obstetrics and Gynecology, West China Second University Hospital, Sichuan University, No. 20, Section 3, Renmin Nan Lu Chengdu, Sichuan 610041, P.R. China

E-mail: sherryxuexiao@yahoo.com

Professor Peng Bai, West China School of Preclinical and Forensic Medicine, Sichuan University, No. 17, Section 3, Renmin Nan Lu Chengdu, Sichuan 610041, P.R. China

E-mail: gynaecologyxx@gmail.com

*Contributed equally

Key words: PSII, NF-кB, proliferation, tumor angiogenesis, VEGF, Bcl-2, Bcl-xL angiogenesis in xenograft athymic mouse models of ovarian cancer (SKOV3 and SKOV3/mutant IkB $\alpha$ cells) using color Doppler ultrasound and traditional immunohistochemistry. We showed that PSII suppressed NF- $\kappa \mathrm{B}$ activation as a result of the reduction in IKK $\beta$ kinase activity on its substrate $\mathrm{I} \kappa \mathrm{B} \alpha$ and the expression of IKK $\beta$. Compromising NF- $\kappa \mathrm{B}$ activation reduced the expression of NF- $\mathrm{B}$-downstream targets such as VEGF, Bcl-2 and Bcl-xL. Such inhibitory effects at molecular levels appear to compromise tumor growth and angiogenesis. Most importantly, the combination of PSII treatment and constitutive repression of IkB $\alpha$ activity exhibited marked inhibitory effects against human ovarian cancer cell growth in a xenograft mouse model of ovarian cancer. For the first time, we provide evidence showing that PSII potently inhibits angiogenesis and the growth of human ovarian cancer by suppressing NF- $\mathrm{NB}$ signaling.

\section{Introduction}

Ovarian cancer is one of the leading causes of cancer-related deaths among women worldwide (1). Poor prognosis is often associated with the advanced stages of ovarian cancer wherein angiogenesis and distant metastases are observed $(2,3)$. Angiogenesis, a key step in tumor growth, can be induced by pro-angiogenic factors such as the fibroblast growth factor, angiopoietin, platelet-derived growth factor and vascular endothelial growth factor (VEGF) (4). VEGF has been shown to support both physiological vasculogenesis and cancer vascular network and is upregulated in many cancer cell types (5). The VEGF family members induce the proliferation, migration and differentiation of endothelial cells, the major component of angiogenesis and lymphogenesis, by binding to VEGF receptor tyrosine kinases on endothelial cells $(6,7)$. The expression of VEGF and the microvessel density are regulated by $N F-\kappa B(8-10)$. NF- $\kappa B$ is an important and ubiquitous transcription factor that often dictates cellular transformation, proliferation, apoptosis, invasion and angio- 
genesis. In the canonical pathway of NF- $\kappa \mathrm{B}$, the activation of an IKK complex phosphorylates I $\mathrm{B}$ proteins that bind and inhibit NF- $\kappa \mathrm{B} /$ Rel proteins. Phosphorylation of I $\mathrm{B}$ subsequently leads to the activation of $\mathrm{NF}-\kappa \mathrm{B} / \mathrm{Rel}$ complexes and their translocation to the nucleus (11). Since the IkB family has been linked to the development of cancer (12), a superengineered repressor of IkB $\alpha$, i.e. IkB $\alpha \mathrm{M}$ (S32A,S36A) was generated (13). IkB $\alpha \mathrm{M}$ has been shown to inhibit the activity of IkB $\alpha$ through blocking the phosphorylation of endogenous $\mathrm{IkB} \alpha$, prevent the translocation of p65 into the nucleus (14-16) and reduce angiogenesis and metastases in ovarian cancer cell lines and other human cancer cell lines $(8,17,18)$.

Rhizoma paridis, a stem of Paris polyphylla Smith var. chinensis (Franch.) Hara or Paris polyphylla Smith var. yunnanensis (Franch.) Hand-Mazz., is known for its many clinical applications in traditional Chinese medicine. Its active components have been used to treat traumatic bleeding, inflammation and microbial infection (19) and most recently, cancer (20). Of the five main active components of Rhizoma paridis (21-23), Paris saponin I (PSI) (polyphyllin D) and Paris saponin II (PSII) (formosanin C), the steroidal saponins, have displayed potent albeit selective cytotoxic effects on tumor cells (24). In our previous study, we demonstrated that PSII suppressed the growth of human ovarian cancer cells via multiple mechanisms including regulation of ERK1/2 activity, promotion cell cycle arrest and activation of the mitochondrial apoptotic pathway (25). We also observed that PSII treatment reduced the expression of NF- $\kappa \mathrm{B}$-downstream targets such as VEGF, Bcl-2 and Bcl-xL. These observations prompted us, in the present study, to examine the possibility that PSII modulates $\mathrm{NF}-\kappa \mathrm{B}$ activity and VEGF-mediated angiogenesis. We also attempted to elucidate the molecular mechanisms underlying such effects in the present study. Our studies revealed that PSII rendered its inhibitory effects by suppressing $\mathrm{NF}-\kappa \mathrm{B}$ signaling in ovarian cancer cells. We also showed that the combination treatment of PSII treatment and the transfection of a super-engineered repressor IKB $\alpha \mathrm{M}$ into SKOV3 cells markedly reduced angiogenesis and tumor growth in xenograft mouse models.

\section{Materials and methods}

Reagents. Purified PSII, isolated from Rhizoma paridis (25), was provided by the Department of Pharmacology at Sichuan University (Chengdu, Sichuan, China). VEGF was obtained from R\&D Systems (Minneapolis, MN, USA). VEGF ELISA kit was purchased from R\&D Systems. Growth factor-reduced Matrigel was from BD Biosciences (San Jose, CA, USA). Antibodies against VEGFR2, VEGF, Bcl-2 and Bcl-xL were obtained from Santa Cruz Biotechnology, Inc. (Santa Cruz, CA, USA). $\beta$-actin antibody was purchased from Sigma Chemical Co. (St. Louis, MO, USA). CD31 antibodies were from Epitomics, Inc. (Burlingame, CA, USA).

Cell lines and cell culture. Primary human umbilical vascular endothelial cells (HUVECs) were from Sichuan University, China. The human high-grade serous ovarian cancer SKOV3 cell line was purchased from the American Type Culture Collection (ATCC; Rockville, MD, USA). SKOV3/vector and SKOV3/I $\kappa \mathrm{B} \alpha \mathrm{M}$ cells were obtained from the University of Texas M.D. Anderson Cancer Center. HUVECs were main- tained in endothelial cell culture medium (ECM) (Lonza, Walkersville, MD, USA) as per the manufacturer's protocol. The SKOV3 cell line was cultured in RPMI-1640 medium (Gibco-BRL, Life Technologies Gaithersburg, MD, USA) supplemented with $10 \%$ fetal bovine serum (FBS) (HyClone, Logan, UT, USA) at $37^{\circ} \mathrm{C}$ under a humidified 95:5\% (v/v) mixture of air and $\mathrm{CO}_{2}$.

Cell viability assay. Cells $\left(5 \times 10^{3}\right.$ cells/well) were directly incubated with indicated concentrations of PSII for indicated durations. Carrier dimethyl sulfoxide (DMSO) $(<0.1 \%)$ was used as a negative control. The cell viability was examined by the 3-[4,5-dimethylthiazol-2-yl]-2,5-diphenyltetrazolium bromide (MTT; Sigma) assay (26). The cytotoxic effects of PSII were expressed as the $50 \%$ inhibitory concentration $\left(\mathrm{IC}_{50}\right)$.

Terminal deoxynucleotidyl transferase-mediated dUTP nick end labeling (TUNEL) assay. The terminal deoxynucleotidyl transferase-mediated dUTP nick end labeling assay was performed as previously described (25). Briefly, cells were treated with PSII after seeding on coverslides for $24 \mathrm{~h}$. All cell layers were fixed by a $4 \%$ paraformaldehyde solution, washed in phosphate-buffered saline (PBS), and then permeated by a permeabilization solution $(0.1 \%$ Triton $\mathrm{X}-100$ solution) for $2 \mathrm{~min}$ at $4^{\circ} \mathrm{C}$. The apoptosis index was determined as the percentage of TUNEL-positive cells/1,000 4,6-diamidino2-phenylindole (DAPI)-stained nuclei. Random fields were recorded using fluorescence microscopy (confocal scanning laser microscopy, Leica TCS4D; Germany).

Endothelial cell Transwell migration assay. The chemotactic motility was carried out using a Transwell migration assay with $8.0-\mu \mathrm{m}$ pore polycarbonate filter inserts as previously described (27). Briefly, the well was coated with $0.1 \%$ gelatin. HUVECs $\left(2 \times 10^{4}\right.$ cells/well) in ECM medium supplemented with $0.5 \%$ FBS were placed in the top chamber. In the bottom chamber, VEGF $(50 \mathrm{ng} / \mathrm{ml})$ in ECM medium was used as a positive control. Conditioned media from SKOV3/vector or $\mathrm{SKOV} 3 / \mathrm{I} \kappa \mathrm{B} \alpha \mathrm{M}$ cells cultured in the presence or absence of PSII were used as a chemoattractant. After $18 \mathrm{~h}$ of incubation, the migrated cells were fixed with $4 \%$ paraformaldehyde and stained with $1 \%$ crystal violet. Images were captured using an Olympus inverted microscope (Olympus; magnification, $\mathrm{x} 200$ ). The migrated cells were counted. Three independent experiments were carried out.

Endothelial cell tube-like network formation assay. A tube formation assay was carried out as previously described (28). Briefly, each well of pre-chilled 24-well plates was coated with $100 \mu \mathrm{l}$ reduced growth factor Matrigel $(2.8 \mathrm{mg} / \mathrm{ml}$; BD Biosciences). Matrigel was polymerized at $37^{\circ} \mathrm{C}$ for 45 min. SKOV3 cells were treated with or without PSII and then incubated with fresh media without PSII for $24 \mathrm{~h}$. The conditioned media were collected and used to study the in vitro tube formation assay. Endothelial cells $\left(1.5 \times 10^{4}\right.$ cells $\left./ \mathrm{ml}\right)$ in ECM medium supplemented with $0.5 \%$ FBS were placed onto the layer of Matrigel. VEGF (50 $\mathrm{ng} / \mathrm{ml}$ ) was used as a positive control for tube formation. After $20 \mathrm{~h}$ of incubation at $37^{\circ} \mathrm{C}$, tubulogenesis was fixed and photographed using a Zeiss microscope equipped with a digital camera and Matrox 
Intellicam imaging software. Tubular structures were quantified using AngioSys software (TCS Cellworks) as per the manufacturer's instructions.

Western blot analysis. The whole-cell lysates were obtained using radioimmunoprecipitation assay (RIPA; Sigma) buffer (20 mmol/1 Tris, $2.5 \mathrm{mmol} / \mathrm{l}$ EDTA, 1\% Triton X-100, 1\% deoxycholate, $0.1 \%$ SDS, $40 \mathrm{mmol} / \mathrm{l} \mathrm{NaF}, 10 \mathrm{mmol} / 1 \mathrm{Na}_{4} \mathrm{P}_{2} \mathrm{O}_{7}$ and $1 \mathrm{mmol} / 1$ phenylmethylsulfonyl fluoride). Protein concentrations were determined using the Bradford assay (Bio-Rad, Hercules, CA, USA) and equalized before loading. Twenty five micrograms of total proteins were resolved using $12 \%$ sodium dodecyl sulfate-polyacrylamide gel electrophoresis (SDS-PAGE). Primary antibodies against Bcl-2 (1:1,000), Bcl-xL $(1: 1,000)$, VEGF $(1: 2,000), \operatorname{I\kappa B} \alpha(1: 1,000)$ p-IкB $\alpha$ (1:1,000, Ser32), phosphorylated P65 (1:2,000, Ser536), P65 $(1: 2,000)$, IккB $(1: 1,000)$ (all from Cell Signaling, Danvers, MA, USA), and HRP-conjugated secondary antibodies were used for the studies. Relative optical density of the protein signals of interest were qualified by ImageJ software (NIH).

Electrophoretic mobility shift assay (EMSA). EMSA study was carried out as previously described (13). Briefly, SKOV3 cells were pretreated with various concentrations of PSII for $24 \mathrm{~h}$. Nuclear extracts were prepared. EMSA was carried out using Ds-Cold-NF- $\mathrm{kB}$ probes: 5'-AGT TGA GGG GAC TT CCC AGG C-3' and 5-TGG GGA ACC TGT GCT GAG TCA CTG GAG-3'. The positive control was the nuclear extracts from the SKOV3 cells treated with $50 \mathrm{ng} / \mathrm{ml} \mathrm{TNF}$ for $30 \mathrm{~min}$. The negative control was the extracts from the SKOV3 cells without any treatment.

Immunoprecipitation (IP) and in vitro kinase assay. The IP and in vitro kinase assays were carried out as previously described (29). Briefly, reaction mixtures $(25 \mu \mathrm{l})$ contained $40 \mathrm{mM} \beta$-glycerophosphate, $7.5 \mathrm{mM} \mathrm{MgCl}_{2}, 5 \%$ glycerol, $7.5 \mathrm{mM}$ EGTA, $\left[\gamma^{-32} \mathrm{P}\right]$ ATP $(0.2 \mathrm{mM}, 1 \mu \mathrm{Ci}), 1 \mathrm{mM}$ orthovanadate, $50 \mathrm{mM} \mathrm{NaF}$ and $0.1 \%(\mathrm{v} / \mathrm{v}) \beta$-mercaptoethanol. The cytoplasmic extract $(2 \mathrm{mg})$ immunoprecipitated with the appropriate antibody was used for the phosphorylation reaction and was washed with lysis buffer containing $50 \mathrm{mM}$ Tris- $\mathrm{HCl}$ (pH 7.5), $120 \mathrm{mM} \mathrm{NaCl}, 5 \mathrm{mM}$ EDTA, $50 \mathrm{mM} \mathrm{NaF}, 0.2 \mathrm{mM}$ $\mathrm{Na}_{3} \mathrm{VO}_{4}, 1 \mathrm{mM}$ DTT, $0.5 \%$ NP-40 and protease inhibitors (Protease Inhibitor Cocktail Tablets; Boehringer, Mannheim; 1 tablet $/ 50 \mathrm{ml}$ ) or with $1 \mu \mathrm{g}$ of purified recombinant GST-IкB $\alpha$ (Cell Signaling) at $37^{\circ} \mathrm{C}$ for $1 \mathrm{~h}$. Reactions were stopped by adding 1 volume of Laemmli sample buffer containing 5\% $\beta$-mercaptoethanol and resolved on a 4-20\% SDS/PAGE gel. Gels were autoradiographed and bands were counted using a Molecular Dynamics PhosphorImager software.

Histology and immunohistochemistry. Paraffin tumor sections (5 $\mu \mathrm{m})$ were derived from solid tumor sections that were resected, fixed with $10 \%$ formaldehyde and embedded in paraffin. Sections were treated with $0.3 \%$ hydrogen peroxide at room temperature to block endogenous peroxidase activities ensued by a $5 \%$ bovine serum albumin incubation. Tumor sections were exposed to antibodies against CD31 (1:100) and VEGFR2 (1:100). Images were captured by a Leica DM 4000B photomicroscope (Solms, Germany; magnification, x200 and $\mathrm{x} 400$ ). The microvessel density was based on CD31 immunohistochemical signals calculated by Image-Pro Plus 6.0 program (Media Cybernetics) $(n=5)$. Hematoxylin and eosin (H\&E) staining was carried out using standard techniques.

Xenograft mouse model of human ovarian tumor. The human ovarian mouse tumor model has been previously described (26). Briefly, 4- to 6-week-old female Balb/c nude mice (Chengdu Experimental Animal Center, Chengdu, China) were randomly divided into 6 groups $(\mathrm{n}=5)$. SKOV3/vector or SKOV3/IкB $\alpha \mathrm{M}$ cells $\left(5 \times 10^{6}\right.$ cells/100 $\left.\mu \mathrm{l}\right)$ were injected subcutaneously into the mice. One week after the implantation, mice were treated with PSII ( 15 and $25 \mathrm{mg} / \mathrm{kg}$ ) by daily intraperitoneal injections. The administrations were carried out on 4 consecutive days/week for 4 weeks (between day 8 and 35). Control groups received the control solution containing the same amount of DMSO $(\mathrm{v}<0.1 \%)$ without PSII. The body weights and tumor volumes were recorded twice weekly. The volume $(\mathrm{V})$ of the solid tumors was measured by a caliber and calculated according to the formula: $\mathrm{V}=$ length $\mathrm{x}$ width ${ }^{2} \mathrm{x} 0.52$. The implanted tumors and vessels were monitored by a Philips HD11 ultrasound scanner (Philips Medical Systems, Best, The Netherlands) equipped with a $11 \mathrm{MHz}$ linear array transducer. The volume of solid tumors (expressed in millimeters) was documented in three dimensions (length, width and height). The minimum diameter of the lesion that can be detected by ultrasound is $0.01 \mathrm{~cm}$. At the termination of the present study, all mice were euthanized using carbon dioxide asphyxiation. All experiments were conducted based on the National Institutes of Health Guidelines for the Care and Use of Experimental Animals. All protocols were approved by the Animal Investigation Committee of the Institute for Nutritional Sciences (Shanghai, China).

Statistical analysis. Statistical significance of differences between groups was performed using one-way ANOVA followed by the Student's t-test. Data are presented as mean \pm standard error (SE). $\mathrm{IC}_{50}$ values were calculated by SPSS software version 13.0 (SPSS, Inc., China). A value of $\mathrm{p} \leq 0.05$ was considered to indicate a statistically significant result.

\section{Results}

PSII treatment inhibits the growth of human tumor cell lines. In our previous study, we demonstrated that PSII had no effects on the survival of non-tumorigenic human vascular smooth muscle, human bronchial or ovarian surface epithelial (OSE) cells. However, PSII decreased the cell viability and inhibited the growth of human tumor cell lines including a high-grade serous ovarian cancer cell line SKOV3 in a concentration-dependent manner (28). Here, we further demonstrated that PSII exhibited more potent antitumorigenic activity than VP16-etoposide. Kinetic studies showed that PSII treatment inhibited SKOV3 cell growth in a concentration-dependent manner. Most important, at the same concentration and at the same time point, PSII killed more cells than VP16, a positive control (Fig. 1). PSII treatment resulted in lower $\mathrm{IC}_{50}$ values (20.99, 10.44, 8.83 and $6.98 \mu \mathrm{M}$, day $1-4$, respectively) than those of VP16 $(82.04,17.18,11.80$ and $8.01 \mu \mathrm{M}$, day $1-4$, respectively). 
SKOV3/Nector

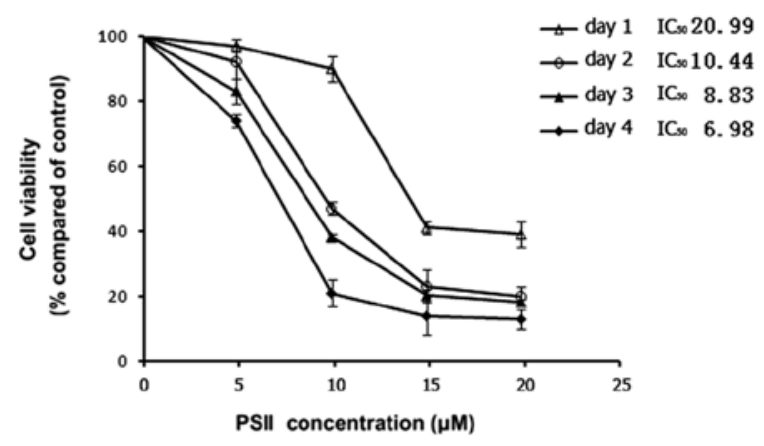

SKOVз/KKaM

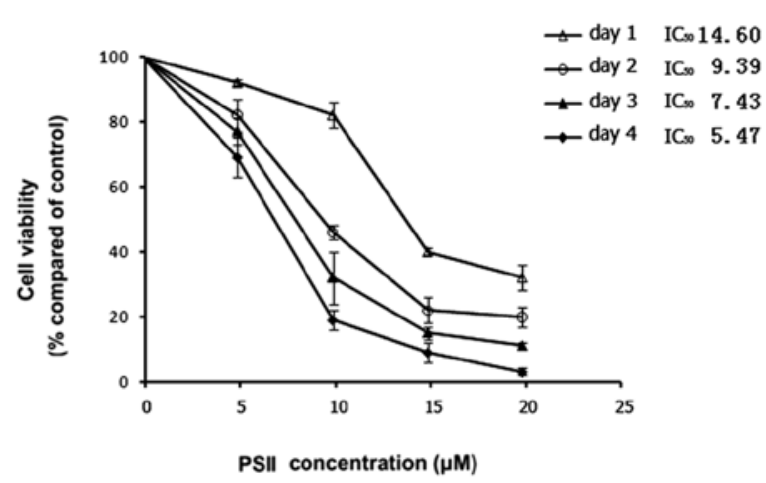

SKOV3/Vector

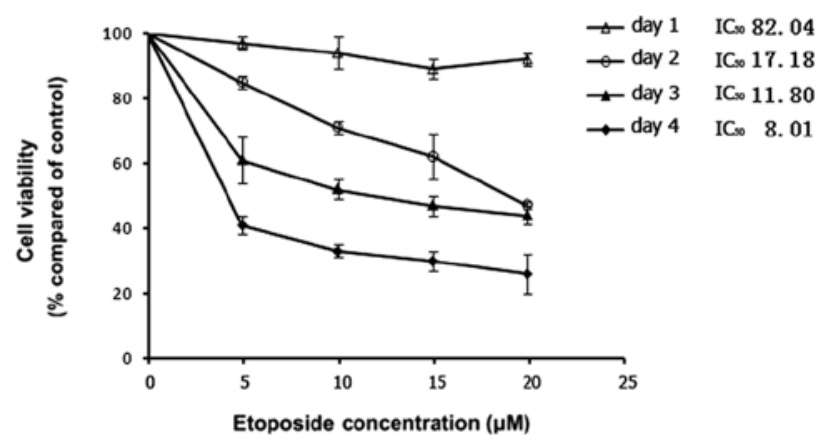

SKOV3/lkBaM

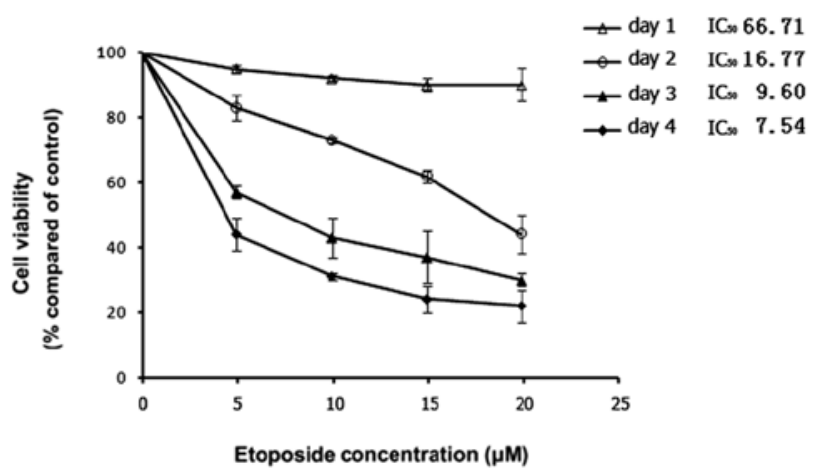

Figure 1. PSII inhibits tumor cell proliferation. SKOV3/vector and SKOV3/I $\mathrm{KB} \alpha \mathrm{M}$ cells were treated with different concentrations of PSII for 1-4 days. Carrier DMSO $(<0.1 \%)$ was used as a negative control. SKOV3/vector and SKOV3/IкB $\alpha$ M cells treated with VP16 (etoposide) were used as positive controls. Cell viability was determined by an MTT assay. IC $_{50}$ values for all treatments are provided in $\mu \mathrm{M}$. p $<0.05, \mathrm{n}=3$. PSII, Paris saponin II; DMSO, dimethyl sulfoxide.

A previous study showed that NF- $\mathrm{KB}$ plays a crucial role in SKOV3 tumor cell growth (13). The transfection of SKOV3 cells with IKB $\alpha \mathrm{M}$ reduced the DNA binding and gene transcription activity of NF- $\mathrm{KB}$. Using this model system, we wanted to further characterize the antitumorigenic property of PSII under a condition wherein the NF- $\kappa \mathrm{B}$ signaling pathway is compromised. Kinetic studies (Fig. 1) demonstrated that the combination treatment (PSII and the transfection of IкB $\alpha \mathrm{M}$ into SKOV3 cells) rendered marked inhibitory effects on tumor cell growth. The antitumorigenic effect was more effective compared to the PSII only treatment. Notably, this combination (PSII and I $\mathrm{B} \alpha \mathrm{M}$ transfection) was more potent than a combination treatment of I $\mathrm{B} \alpha \mathrm{M}$ transfection and VP16, an antimicrotubule agent (our positive control). For example, the maximum inhibitory ratio achieved with $10 \mu \mathrm{M}$ PSII treatment and IкB $\alpha \mathrm{M}$ transfection following a 4-day treatment was $82 \%$ compared with the $65 \%$ inhibition produced by $10 \mu \mathrm{M}$ VP16 treatment and I $\kappa \mathrm{B} \alpha \mathrm{M}$ transfection. In this model, PSII treatments also yielded lower $\mathrm{IC}_{50}$ values compared to those of the VP16 treatments. Since our interest focused on the effects of PSII on NF- $\kappa B$ activity, VEGF-mediated angiogenesis, and the molecular mechanisms underlying such effects but not cell death, the above studies allowed us to identify the sub-cytotoxic concentrations of PSII to be used for most of the subsequent studies $(2.5$ and $5 \mu \mathrm{M})$.

PSII inhibits SKOV3 cell-induced HUVEC motility and tube-like network formation. Cell motility and tubulogenesis are crucial steps in angiogenesis (30). Therefore, we wanted to determine whether PSII treatment affects the ability of tumor cells to induce endothelial cell migration and tube formation. First, using the Boyden chamber assay, we showed that conditioned media from the PSII-treated SKOV3 cells failed to induce HUVEC migration as compared to the control-treated group (carrier DMSO, <0.1\%) and the VEGF-induced migration group (Fig. 2A and B). We further demonstrated that PSII treatment also modulated SKOV3 cell-induced tubulogenesis. As shown in Fig. 2D, conditioned media from the PSII-treated SKOV3 cells also failed to induce tube formation in a concentration-dependent manner compared to the control treatment (VEGF only, $50 \mathrm{ng} / \mathrm{ml}$; and DMSO carrier, $<0.1 \%$ ). The disruption of tube formation was observed in HUVECs following treatment with cytotoxic levels of PSII $(2.5 \mu \mathrm{M})(28)$. We also measured the VEGF level in the conditioned medium obtained from the PSII-treated SKOV3 cells. A marked reduction in VEGF levels was observed in the conditioned media of the PSII-treated SKOV3 cells compared to the control (Fig. 2C). We further examined the possibility that the reduced VEGF level may be attributed to cell death. As shown in Fig. 3C, TUNEL staining studies showed a minimal increase in the percentage of TUNEL-positive SKOV3 cells following treatment with $2.5 \mu \mathrm{M}$ PSII compared to the control. Together, the results suggest that PSII treatment modulates VEGF levels in the tumor microenvironment leading to reduced capillary formation.

PSII inhibits the activity of $N F-\kappa B$ in human ovarian cancer cells. Since a previous study (13) demonstrated that $\mathrm{NF}-\kappa \mathrm{B}$ 

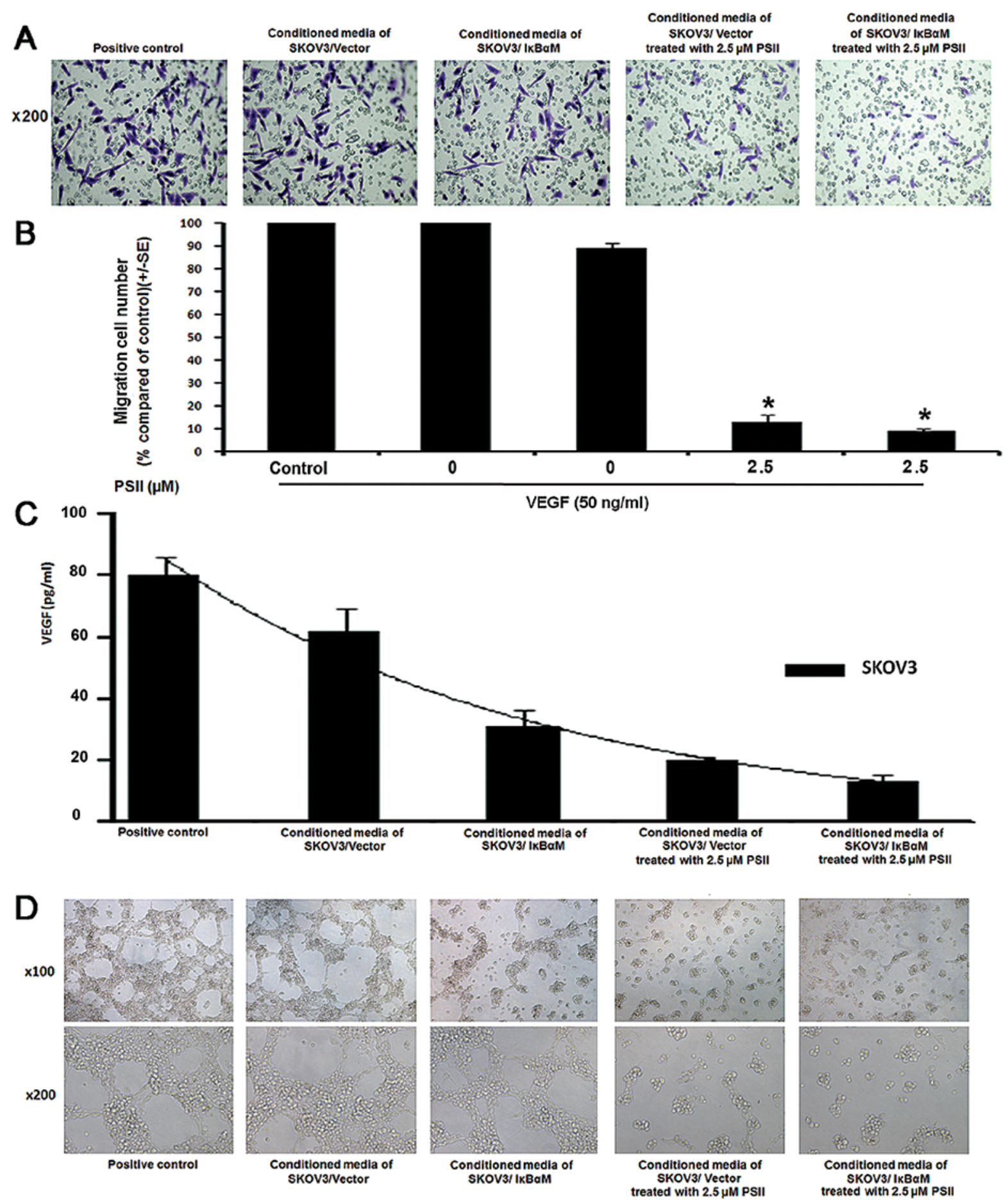

Figure 2. PSII inhibits tumor cell-induced HUVEC cell migration and tubulogenesis in Matrigel matrix. (A) Representative images depicting that PSII treatment prevents SKOV3/vector or SKOV3/IKB $\alpha$ M cells to induce HUVEC cell migration. A cell motility study was carried out using a Transwell chamber system and conditioned media from SKOV3/vector or SKOV3/IкB $\alpha \mathrm{M}$ cells. HUVECs were seeded in the upper chamber. In the lower chamber, conditioned media from PSII treated SKOV3/vector or SKOV3/IкB $\alpha \mathrm{M}$ cells were used as chemoattractants. VEGF was used as a positive control. Images were captured using an inverted microscope at magnification, $\mathrm{x} 200$. Carrier DMSO $(<0.1 \%)$ was used as a negative control. (B) Data represent the mean \pm standard error of the mean (SEM) percentage of the number of cells migrating to the lower chamber compared to the control (VEGF); ${ }^{*}<0.05$, $n=3$. (C) Concentration of VEGF protein in conditioned medium. PSII $(2.5 \mu \mathrm{M})$-treated or non-treated SKOV3/vector or SKOV3/IкB $\alpha \mathrm{M}$ cells were cultured in fresh serum-free medium. Culture supernatants (conditioned media) were collected and VEGF protein levels were measured by ELISA. Each value represents the mean of three independent experiments; bars, \pm SD. (D) Representative images showing the tube formation network induced by VEGF (positive control) or conditioned media from PSII $(2.5 \mu \mathrm{M})$-treated or non-treated SKOV3/vector or SKOV3/IkB $\alpha$ M cells. Bright-field images were recorded at x200/100 magnification and processed for analysis. PSII, Paris saponin II; DMSO, dimethyl sulfoxide.

plays an important role in SKOV3 tumor cell growth and PSII treatment affects the levels of SKOV3-secreted VEGF, a target of the NF- $\kappa B$ signaling pathway (31), we wanted to examine whether PSII modulates NF- $\kappa \mathrm{B}$ activity in SKOV3 cells. In Fig. 3A, EMSA data indicated that the binding of NF- $\mathrm{KB}$ to its promoter DNA consensus sequence was reduced in the PSII- 
A

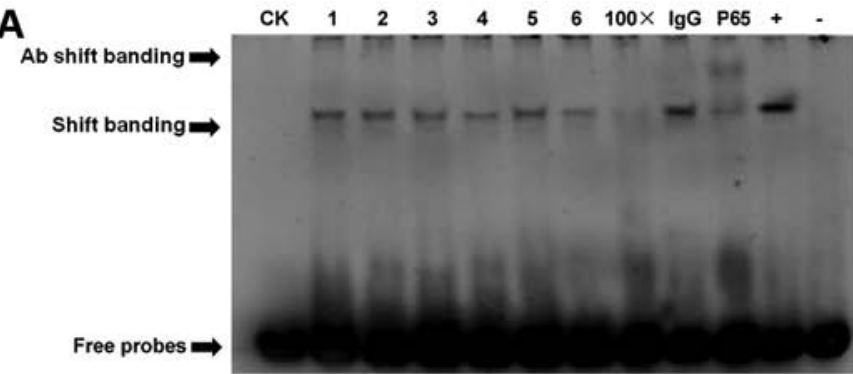

C
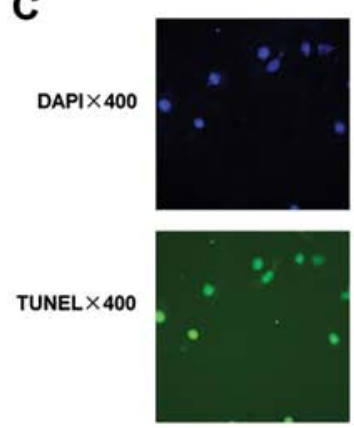

SKov3/vector
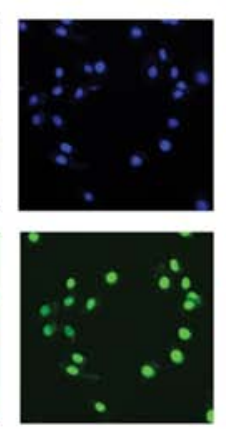
SKOV3/vector treated
with $2.5 \mu \mathrm{M}$ PSII
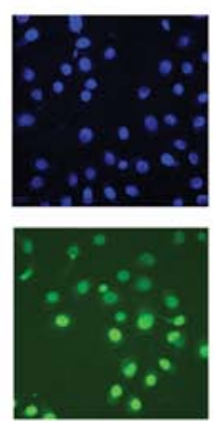

SKOV $3 /$ KBaM treated
with $2.5 \mu M$ PSII
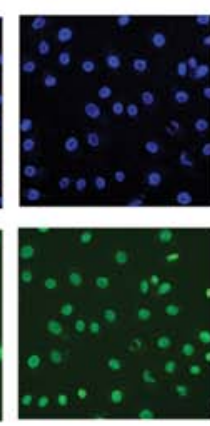

SKOV3/vector treat with $5 \mu \mathrm{M}$ PSII
1 SKov3/Vector treated DMSO 2 SKOV $3 /$ Vector treated with $1 \mu \mathrm{M}$ PSII 3 SKOV $3 /$ Vector treated with $2.5 \mu \mathrm{M}$ PSI 4 SKOV3/Vector treated with $5 \mu \mathrm{M}$ PSII 5 SKOV3/Vector 6 SKOVз/IKBaM

Figure 3. PSII modulates NF- $\kappa$ B activity and the expression of the downstream targets of NF- $\kappa$ B. (A) The electrophoretic mobility shift assay shows the reduced binding of NF- $\kappa \mathrm{B}$ to its promoter DNA consensus sequence in the PSII-treated SKOV3 cells. Nuclear extracts from SKOV3 cells treated with 50 ng/ml TNF for $30 \mathrm{~min}$ were used as a positive control. The negative controls were the nuclear extracts from the SKOV3 cells without any treatment. (B) Western blot analysis of cells treated with different concentrations of PSII for $24 \mathrm{~h}$. PSII treatment modulated anti-apoptotic proteins, NF- $\kappa \mathrm{B}-\mathrm{p} 65$ and VEGF expression levels in the

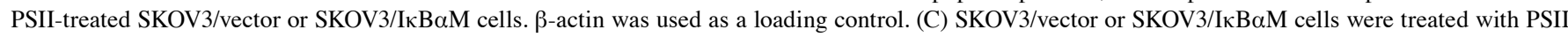
$(2.5$ and $5 \mu \mathrm{M})$ and labeled with TUNEL and DAPI after $24 \mathrm{~h}$ of PSII treatment. Images of random fields $(\mathrm{n}=10) / \mathrm{slide}(\mathrm{n}=3) \mathrm{were}$ collected at $\mathrm{x} 400 \mathrm{for}$ data analysis. Data are presented as the percentage of TUNEL-positive cells (green fluorescence)/total number of treated cells (blue DAPI staining). Representative figures of treated cells labeled for TUNEL and counterstained with DAPI are shown. PSII, Paris saponin II.
A

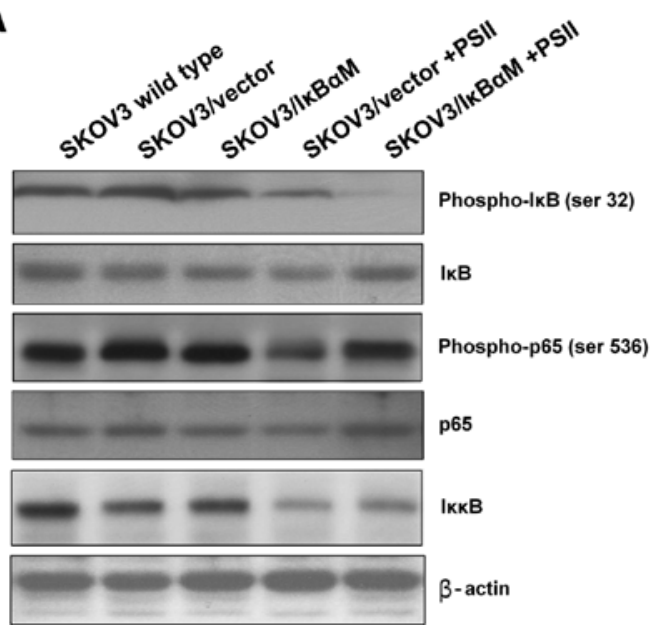

B

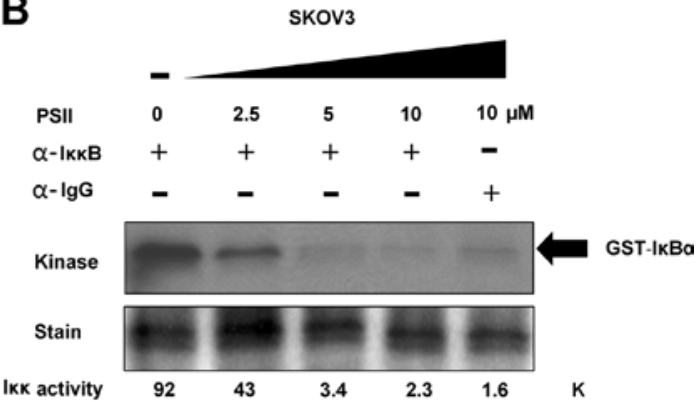

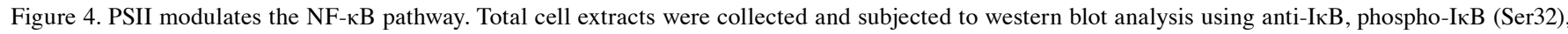
p65, phosphor-p65 (Ser536), IKK $\beta$ and actin. Twenty five micrograms of extract from SKOV3 wild-type, SKOV3/vector, SKOV3/IKB $\alpha \mathrm{M}$, SKOV3/vector cells treated with $2.5 \mu \mathrm{M}$ PSII and SKOV3/IкB $\alpha \mathrm{M}$ cells treated with $2.5 \mu \mathrm{M}$ PSII were separated using SDS/PAGE. (B) PSII reduced the IKK $\beta$ activity in the SKOV3 cells. An equal amount $(2 \mathrm{mg})$ of cytoplasmic proteins from the SKOV3 cells treated with PSII (concentrations) was immunoprecipitated with the anti-

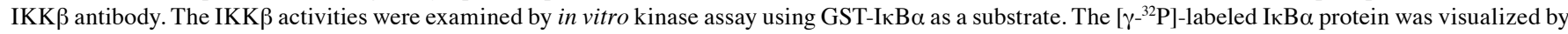
autoradiography. The IKK $\beta$ activities were quantitated by ImageQuant software. The bottom panel shows Coomassie staining of GST-IкB $\alpha$ to show the equal amount of substrate in each reaction. PSII, Paris saponin II.

treated cells at a concentration as low as $2.5 \mu \mathrm{M}$. Notably, PSII treatment $(5 \mu \mathrm{M})$ and the transfection of $\mathrm{I} \kappa \mathrm{B} \alpha \mathrm{M}$ into SKOV3 cells yielded a comparable reduction in DNA binding. Such observable effects of PSII on NF- $\kappa$ B transcriptional activity led us to examine potential changes in the expression of downstream $\mathrm{NF}-\kappa \mathrm{B}$ targets. In Fig. $3 \mathrm{~B}$, we showed that PSII treatment modulated the expression of well-known NF- $\kappa \mathrm{B}$ targets, e.g. Bcl-2, Bcl-xL and VEGF (31). PSII treatment led to the downregulation of these targets in a concentration-dependent manner. This finding supports the previous experiment showing the reduced levels of secreted VEGF in the conditioned media of PSII-treated cells (Fig. 2C). Together, the results indicated 
A

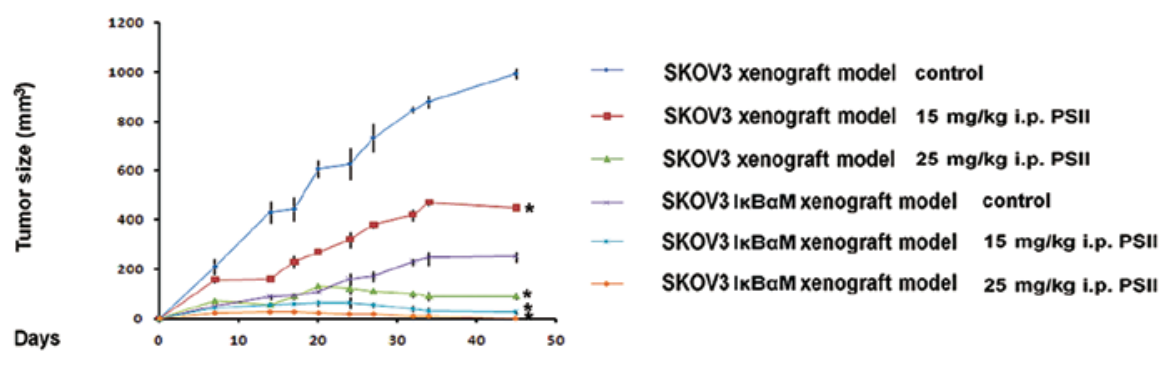

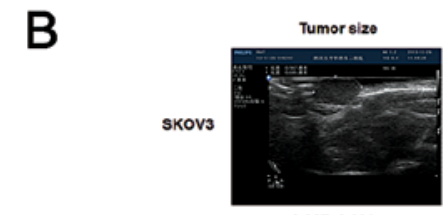

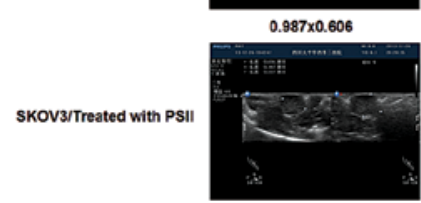

$0.696 \times 0.487$

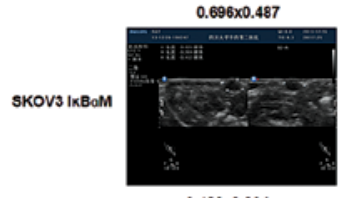

$0.426 \times 0.304$

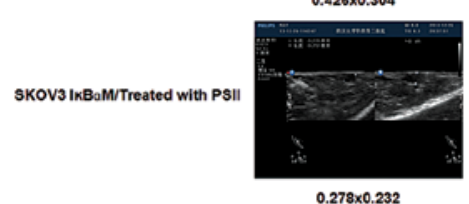

$0.278 \times 0.232$

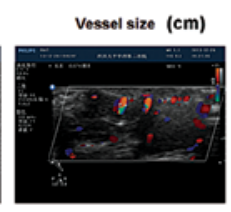

0.074

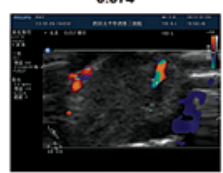

0.057

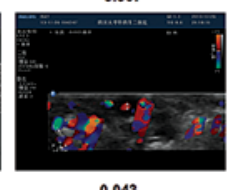

0.043

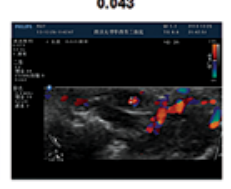

0.043

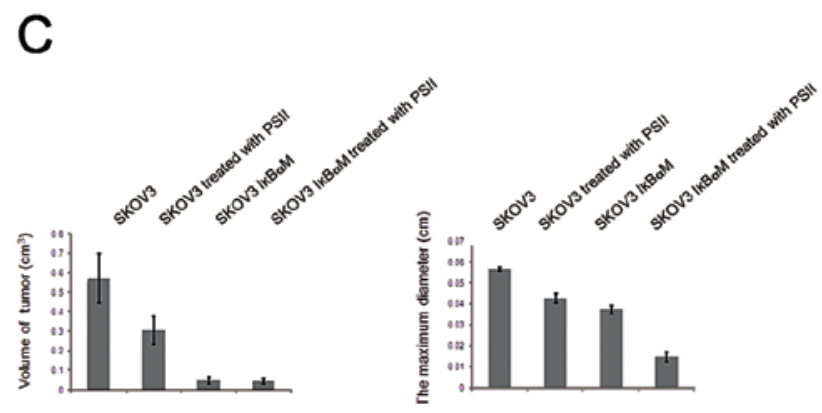

E
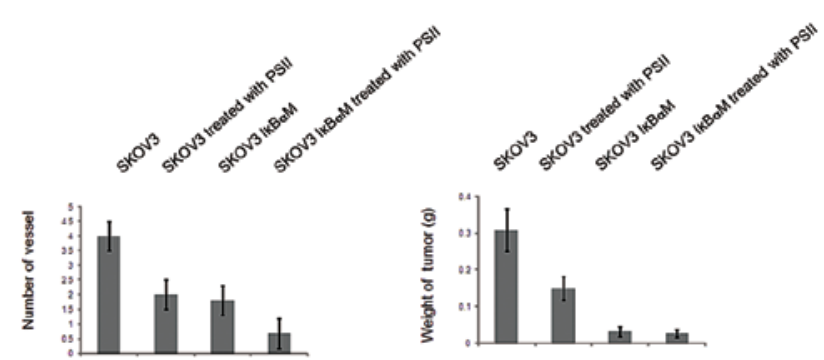

Figure 5. PSII suppresses tumor growth and angiogenesis in a xenograft mouse model of ovarian cancer. (A) Mice were implanted with $5 \times 10^{6} \mathrm{SKOV} 3 / \mathrm{vector}$ or SKOV3/IKB $\alpha M$ cells on day 0 and were randomly divided into various treatment and control groups $(\mathrm{n}=5)$. Eight days after the implantation, the tumor-bearing mice were treated according to the protocols. Briefly, tumor-bearing mice were treated with PSII or received the vehicle (DMSO, $<0.1 \%$ ) in saline solution by intraperitoneal administration for 4 weeks, 4 consecutive days/week with two different doses of PSII, $15 \mathrm{or} 25 \mathrm{mg} / \mathrm{kg}$. Columns, mean; bars, SD, ${ }^{*} \mathrm{p}<0.05$, compared to the control. (B) Implanted tumors, delined by the yellow lines, and vessels in the PSII-treated and control mice were monitored by a Philips HD11 ultrasound scanner. The flows toward and away from the ultrasound transducer were noted by the red and blue color, respectively. (C) Measurement of the tumor wet weight, tumor volume, the maximum diameter of blood vessels and the number of vessels are presented. Columns, mean ( $\mathrm{n}=3$ ); bars, SE. PSII, Paris saponin II.

that PSII treatment compromises NF- $\kappa \mathrm{B}$ activation and reduces the expression of several $\mathrm{NF}-\kappa \mathrm{B}$-downstream targets known to play pivotal roles in tumor growth biology.

Effect of PSII on components of the NF- $\kappa$ B canonical pathway. Since the binding of $N F-\kappa B$ to its promoter DNA consensus sequence and the expression of several NF- $\kappa \mathrm{B}$-downstream targets were altered by the treatment of PSII, we wanted to determine whether I $\kappa \mathrm{B} \alpha$ or P65 levels were altered in PSIItreated cells. Protein signals of I $\kappa \mathrm{B} \alpha$, phosphorylated-I $\kappa \mathrm{B} \alpha$, P65, phosphorylated-P65 and IKK $\beta$ were assessed. Protein signals from the extracts of SKOV3, SKOV3/vector and $\mathrm{SKOV} 3 / \mathrm{I} \kappa \mathrm{B} \alpha \mathrm{M}$ cells were used as controls. The results from Fig. 4A showed that while there was no change in the total $\mathrm{I} \kappa \mathrm{B} \alpha$ and P65 levels in all conditions, unexpectedly, PSII treatment $(2.5 \mu \mathrm{M})$ appeared to reduce IKK $\beta$ expression. Consistent with this observation, PSII treatment also reduced phosphorylation of $\mathrm{I} \kappa \mathrm{B} \alpha$ and P65 on Ser32 and Ser536, respectively.

Next, we examined whether PSII treatment also affects IKK $\beta$ kinase activity on its substrate I $\mathrm{B} \alpha$. Immunoprecipitated IKK $\beta$ from the extracts of the PSII-SKOV3 treated cells and carrier DMSO-treated cells were used in an in vitro kinase assays. Fig. 4B showed that control cells had stronger IKK $\beta$ kinase activity as compared to that of the PSII-treated SKOV3 cells. Indeed, PSII treatment suppressed IKK $\beta$ activity in a concentration-dependent manner. Together, these results indicate that PSII targets IKK $\beta$ leading to a reduction in $N F-\kappa B$ signaling and the expression of NF- $\mathrm{KB}$-downstream targets.

PSII and mutant IkB $\alpha$ treatment inhibit tumor growth and angiogenesis in a xenograft mouse model of human ovarian cancer. Our previous study showed that PSII suppresses tumor growth in a xenograft mouse model of ovarian cancer (25). To characterize the anti-angiogenic property of PSII in vivo and the relationship with NF- $\kappa \mathrm{B}$ signaling pathway, we continued to use this xenograft model employing SKOV3/vector and SKOV3/IkB $\alpha \mathrm{M}$ cells. Consistent with our previous finding (28), PSII treatments suppressed tumor growth rates and reduced the tumor weights and tumor sizes as compared to the control treatment (Fig. 5A and C). In our previous study, we demonstrated that color Doppler ultrasound can be used as a non-invasive method to assess angiogenesis (28). Using this approach (Fig. 5B), here, we confirmed that, on day 35, before the termination of the in vivo tumor growth study, PSII rendered 

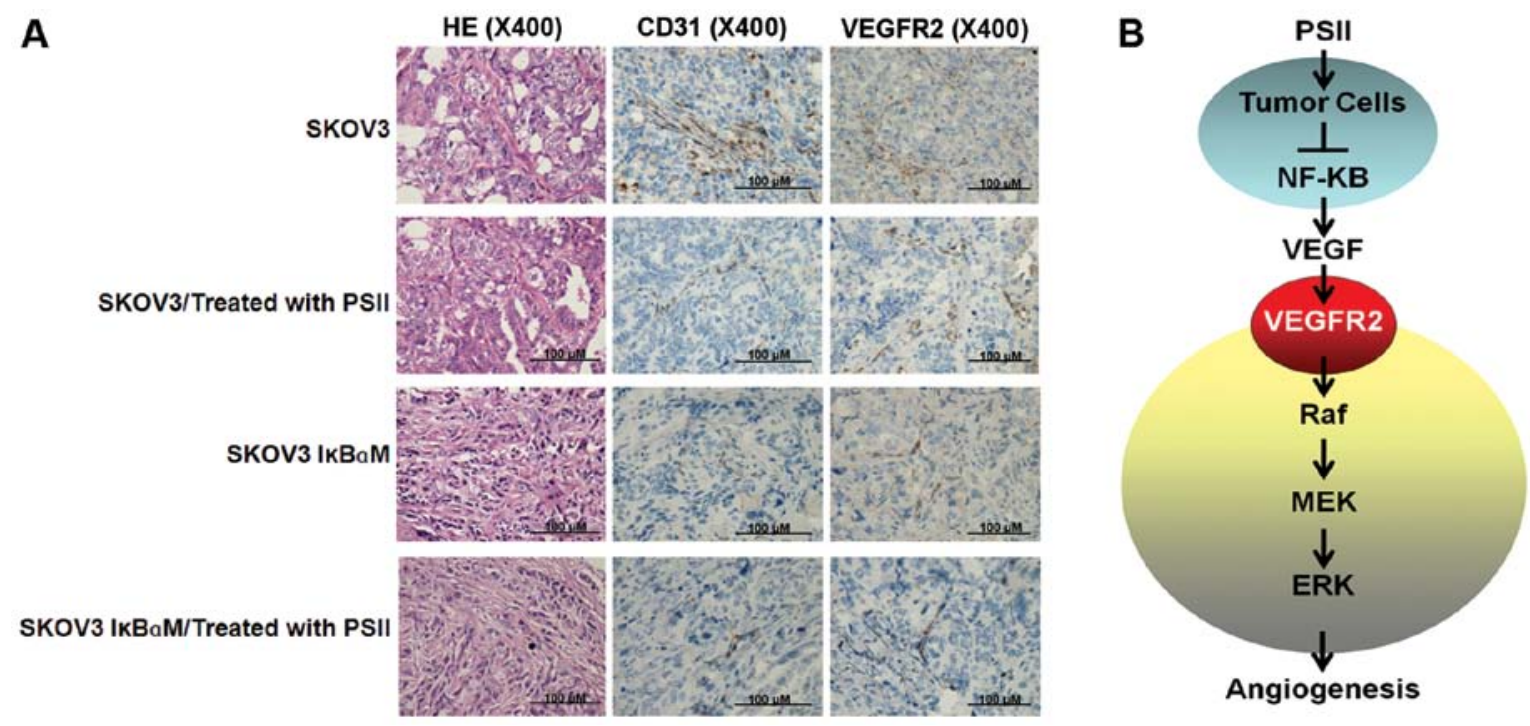

Figure 6. PSII inhibits angiogenesis. (A) Representative images of random mouse tumor sections of the grafts of SKOV3 and SKOV3/IKB $\alpha \mathrm{M}$ cells treated with PSII (15 mg/kg) or control (no treatment) are shown. Sections were labeled with blood vessel markers CD31 and VGFR2. Images were captured at the magnification of $x 400$. Vessel staining further confirmed the results of the Doppler ultrasound confirming that PSII treatment inhibits neovascularization. (B) Schematic model depicts the PSII-mediated anti-angiogenic effects. PSII modulates the tumor microenvironment by altering VEGF production released from tumor and endothelial cells. PSII treatment inhibits NF- $\mathrm{KB}$ activation in tumor cells leading to the reduction of several downstream NF- $\mathrm{kB}$ targets including VEGF and pro-apoptotic proteins. PSII, Paris saponin II.

profound inhibitory effects on neovascularity reflected by the stark reduction in spectral and color Doppler signals in the PSII-treated groups compared to that of the control mice. The maximum diameter of blood vessels and microvessel density were significantly reduced $(\mathrm{p}<0.05)$ (Fig. 5C). The aforementioned reductions were also correlated with the suppression of tumor growth evident by a $>50 \%$ reduction in tumor wet weights as compared to that of the controls $(\mathrm{p}<0.05)$ (Fig. 5C).

Notably, in combination with the transfection of the super-engineered repressor of ІкB $\alpha$, i.e. IкB $\alpha \mathrm{M}$ (S32A and S36A) known to inhibit NF-kB activity, the antitumorigenic effects of PSII were enhanced significantly. While the effects of PSII treatment alone or in combination with IKB $\alpha \mathrm{M}$ rendered similar inhibitory effects on tumor wet weights and tumor volumes, the combination treatment markedly suppressed the growth rate and reduced the number of vessels and the maximum diameter of blood vessels (by $>50 \%$ ) compared to that of the PSII only treatment (Fig. 5C). Clearly, the combination treatment exerts marked and extremely effective anti-angiogenic effects. To confirm this observation, we used immunohistochemistry to assess signals of angiogenesis markers, i.e. VEGFR2 and CD31, on tumor sections of the grafts from the treated or control mice. Our results confirmed that PSII treatment clearly reduced the expression of VEGFR2, the key player of VEGF signaling and microvessel density reflecting by CD31 signals (Fig. 6A). These data also suggest the possibility of using PSII in combination with a drug that inhibits NF-KB signaling. Together, the data in the present study allowed us to elucidate a novel antitumorigenic and anti-angiogenic feature of PSII (Fig. 6B). For the first time, we provide evidence showing that PSII potently inhibits angiogenesis and the growth of human ovarian cancer by suppressing NF- $\mathrm{KB}$ signaling in cancer cells leading to the suppression of pro-angiogenic factors of the tumor microenvironment, e.g. VEGF.

\section{Discussion}

Saponin II (formosanin C), a steroidal saponin, is one of the main active components of Rhizoma paridis $(21-23,32)$. In our previous study, we characterized the antitumorigenic and antiangiogenic effects of PSII in a mouse model of ovarian cancer. We showed that PSII not only induced tumor cell death but also compromised endothelial cell activity leading to inhibitory effects on angiogenesis in different in vivo, ex vivo and in vitro model systems of angiogenesis (25). In the present study, we demonstrated that PSII also modulated angiogenesis indirectly by targeting VEGF expression in tumor cells. Specifically, PSII reduced IKK $\beta$ expression and reduced its kinase activity. While the reduction of IKK $\beta$ kinase activity can be attributed to the degradation of IKK $\beta$ upon PSII treatment, most importantly, this degradation did affect downstream expression of anti-apoptotic molecules and pro-angiogenic VEGF in tumor cells. Our results were also consistent with previous studies showing that NF- $\mathrm{KB}$ regulated VEGF expression and the microvessel density (8-10).

The binding of VEGF to its receptors causes receptor dimerization and auto-phosphorylation leading to the activation of several downstream kinases and the expression of anti-apoptotic proteins such as Bcl-2. The expression of Bcl-2 in tumor-associated endothelial cells could be induced by VEGF secreted from tumor cells and endothelial cells in the tumor microenvironment (33). Studies have shown that the upregulation of expression of Bcl-2 in microvascular endothelial cells could also promote intratumoral angiogenesis and tumor growth $(34,35)$. Therefore, targeting the VEGF signaling pathway in either tumor cells or endothelial cells may compromise angiogenesis. As shown in the present study, PSII reduced VEGF expression and tumor cell-secreted VEGF levels at a subcytotoxic level. Nevertheless, at such a concentration, PSII still reduced NF- $\mathrm{kB}$ activities in tumor 
cells resulting in low VEGF levels in the tumor microenvironment. The result demonstrated the therapeutic potential of PSII in anti-angiogenic therapy. PSII, at low-doses, prevented tumor-induced angiogenesis without damaging the healthy endothelial cells. At such low doses, patients may avoid side-effects often observed in anti-angiogenic therapy (36).

Our previous study demonstrated that PSII exhibited antitumorigenic ability in human ovarian cancer cells by inducing apoptosis (25). Here, we confirmed that such induction was the result of the PSII treatment. PSII treatment altered the expression of anti-apoptotic proteins Bcl-2 and Bcl-xL in the SKOV3 cell line in a concentration-dependent manner. Notably, such downregulation was even more prominent when the NF- $\mathrm{\kappa B}$ activation in the SKOV3 cells transfected with IкB $\alpha$ M was compromised. Our results were consistent with previous studies showing that suppression of the NF- $\mathrm{KB}$ activation could be attributable to increased levels of apoptosis (8).

In conclusion, in the present study, for the first time, we identified molecular targets of a steroidal saponin family member, PSII. PSII modulated IKK $\beta$ expression and kinase activity leading to a reduction in NF- $\mathrm{KB}$ transactivation. As a result, the treatment altered the expression of several downstream targets of NF- $\kappa \mathrm{B}$, i.e. VEGF, Bcl-2 and Bcl-xL. Most importantly, we continued to demonstrate the therapeutic potential of a combination treatment using PSII. PSII can be used in combination with other drugs/agents that modulate $\mathrm{NF}-\mathrm{\kappa B}$ transactivation in cancer cells.

\section{Acknowledgements}

The present study was supported by the Fund of the National Nature Science Foundations grant no. 81001159/81202387 and the Sichuan Province Science and Technology Plan Project no. 2013JY0013/2014JY0213 and the Scientific Research Foundation of Sichuan University for outstanding young scholars no. 2013SCU04A22. The authors would like to thank Dr Meifang Xiao and Dr Hong Zou for providing us with the PSI and PSII compounds, and also like to thank Diane Hackett and Maude E. Veech for their technical support.

\section{References}

1. Siegel R, Naishadham D and Jemal A: Cancer statistics, 2013. CA Cancer J Clin 63: 11-30, 2013.

2. McGuire WP, Hoskins WJ, Brady MF, et al: Cyclophosphamide and cisplatin compared with paclitaxel and cisplatin in patients with stage III and stage IV ovarian cancer. $\mathrm{N}$ Engl J Med 334: $1-6,1996$.

3. Eckstein N: Platinum resistance in breast and ovarian cancer cell lines. J Exp Clin Cancer Res 30: 91, 2011.

4. Carmeliet P: Angiogenesis in life, disease and medicine. Nature 438: 932-936, 2005.

5. Kerbel RS: Tumor angiogenesis. N Engl J Med 358: 2039-2049, 2008.

6. Xu W, Liu LZ, Loizidou M, Ahmed M and Charles IG: The role of nitric oxide in cancer. Cell Res 12: 311-320, 2002.

7. Ferrara $\mathrm{N}$ and Kerbel RS: Angiogenesis as a therapeutic target. Nature 438: 967-974, 2005

8. Huang S, Robinson JB, Deguzman A, Bucana CD and Fidler IJ: Blockade of nuclear factor- $\kappa \mathrm{B}$ signaling inhibits angiogenesis and tumorigenicity of human ovarian cancer cells by suppressing expression of vascular endothelial growth factor and interleukin 8. Cancer Res 60: 5334-5339, 2000.

9. Aggarwal BB: Nuclear factor-kappaB: the enemy within. Cancer Cell 6: 203-208, 2004.
10. Yu HG, Yu LL, Yang Y, et al: Increased expression of RelA/ nuclear factor- $\mathrm{\kappa B}$ protein correlates with colorectal tumorigenesis. Oncology 65: 37-45, 2003.

11. Biswas DK, Shi Q, Baily S, et al: NF- $\kappa \mathrm{B}$ activation in human breast cancer specimens and its role in cell proliferation and apoptosis. Proc Natl Acad Sci USA 101: 10137-10142, 2004.

12. Gilmore TD,KoedoodM,PiffatKA and WhiteDW:Rel/NF-kappaB/ IkappaB proteins and cancer. Oncogene 13: 1367-1378, 1996.

13. Yang G, Xiao X, Rosen DG, et al: The biphasic role of NF- $\kappa B$ in progression and chemoresistance of ovarian cancer. Clin Cancer Res 17: 2181-2194, 2011.

14. Scherer DC, Brockman JA, Chen Z, Maniatis T and Ballard DW: Signal-induced degradation of $\mathrm{I} \kappa \mathrm{B} \alpha$ requires site-specific ubiquitination. Proc Natl Acad Sci USA 92: 11259-11263, 1995.

15. Brown K, Gerstberger S, Carlson L, Franzoso G and Siebenlist U: Control of I kappa B-alpha proteolysis by site-specific, signalinduced phosphorylation. Science 267: 1485-1488, 1995.

16. Brockman JA, Scherer DC, McKinsey TA, et al: Coupling of a signal response domain in I $\mathrm{B} \alpha$ to multiple pathways for NF- $\kappa \mathrm{B}$ activation. Mol Cell Biol 15: 2809-2818, 1995.

17. Huang S, Pettaway CA UH, Bucana CD and Fidler IJ: Blockade of NF- $\kappa \mathrm{B}$ activity in human prostate cancer cells is associated with suppression of angiogenesis, invasion, and metastasis. Oncogene 20: 4188-4197, 2001.

18. Fujioka S, Sclabas GM, Schmidt C, et al: Function of nuclear factor $\kappa \mathrm{B}$ in pancreatic cancer metastasis. Clin Cancer Res 9: 346-354, 2003.

19. Ho JW, Leung YK and Chan CP: Herbal medicine in the treatment of cancer. Curr Med Chem Anticancer Agents 2: 209-214, 2002.

20. Man S, Gao W, Zhang Y, et al: Antitumor and antimetastatic activities of Rhizoma Paridis saponins. Steroids 74: 1051-1056, 2009.

21. Cosgrove D: Angiogenesis imaging - ultrasound. Br J Radiol 76 (Spec No 1): S43-S49, 2003.

22. Howard-Claudio C: MRI methods for the detection of angiogenesis. Supplement to Appl Radiol: 34, 2005.

23 Man S, Gao W, Zhang Y, et al: Characterization of steroidal saponins in saponin extract from Paris polyphylla by liquid chromatography tandem multi-stage mass spectrometry. Anal Bioanal Chem 395: 495-505, 2009.

24. Xiao X, Bai P, Bui Nguyen TM, et al: The antitumoral effect of Paris Saponin I associated with the induction of apoptosis through the mitochondrial pathway. Mol Cancer Ther 8: 1179-1188, 2009.

25. Xiao X, Zou J, Bui-Nguyen TM, et al: Paris saponin II of Rhizoma Paridis - a novel inducer of apoptosis in human ovarian cancer cells. Biosci Trends 6: 201-211, 2012.

26. Schmitt J and Matei D: Targeting angiogenesis in ovarian cancer. Cancer Treat Rev 38: 272-283, 2012.

27. Nicosia RF and Ottinetti A: Growth of microvessels in serum-free matrix culture of rat aorta. A quantitative assay of angiogenesis in vitro. Lab Invest 63: 115-122, 1990.

28. Xiao X, Yang M, Xiao J, et al: Paris Saponin II suppresses the growth of human ovarian cancer xenografts via modulating VEGF-mediated angiogenesis and tumor cell migration. Cancer Chemother Pharmacol 73: 807-818, 2014.

29. Agbottah E, Yeh WI, Berro R, et al: Two specific drugs, BMS-345541 and purvalanol A induce apoptosis of HTLV-1 infected cells through inhibition of the NF-kappaB and cell cycle pathways. AIDS Res Ther 5: 12, 2008.

30. Patan S: Vasculogenesis and angiogenesis. Cancer Treat Res 117: 3-32, 2004.

31. Fujioka S, Sclabas GM, Schmidt C, et al: Inhibition of constitutive NF- $\kappa \mathrm{B}$ activity by $\mathrm{I} \kappa \mathrm{B} \alpha \mathrm{M}$ suppresses tumorigenesis. Oncogene 22: 1365-1370, 2003.

32. Wen F, Yin H, Chen C, et al: Chemical characteristics of saponins from Paris fargesii var. brevipetala and cytotoxic activity of its main ingredient, paris saponin H. Fitoterapia 83: 627-635, 2012.

33. Nor JE, Christensen J, Mooney DJ and Polverini PJ: Vascular endothelial growth factor (VEGF)-mediated angiogenesis is associated with enhanced endothelial cell survival and induction of Bcl-2 expression. Am J Pathol 154: 375-384, 1999.

34. Nör JE, Christensen J, Liu J, et al: Up-Regulation of Bcl-2 in microvascular endothelial cells enhances intratumoral angiogenesis and accelerates tumor growth. Cancer Res 61: 2183-2188, 2001.

35. Karl E, Warner K, Zeitlin B, et al: Bcl-2 acts in a proangiogenic signaling pathway through nuclear factor- $\kappa \mathrm{B}$ and $\mathrm{CXC}$ chemokines. Cancer Res 65: 5063-5069, 2005.

36. Lacouture ME,Lenihan DJ and Quaggin SE (eds): Antiangiogenic Therapy: Tolerability and Management of Side Effects, 2009. (www.angio.org/pdf/Angio_Poster_Final_6-30.pdf). 